\title{
Prognostic Value of Three Rapid Scoring Scales and Combined Score for the Assessment of Patients with Coronavirus Disease 2019
}

Hai Hu

Sichuan University West China Hospital

Weili Kong

Sichuan University West China Hospital https://orcid.org/0000-0002-1248-5522

Ni Yao

Sichuan University West China Hospital

Yanru Qiu

Wuhan University Renmin Hospital

Hailing Gu

Sichuan University West China Hospital

Wei Xu ( $\nabla$ wei.xu@uhnresearch.ca )

Research

Keywords: COVID-19; Risk factors; Prognosis; Prediction.

Posted Date: April 29th, 2020

DOl: https://doi.org/10.21203/rs.3.rs-25147/v1

License: (9) (1) This work is licensed under a Creative Commons Attribution 4.0 International License. Read Full License 


\section{Abstract}

Background: Severe coronavirus disease 2019 (COVID-19) is associated with high mortality, whereas mild cases generally have a good prognosis. Therefore, the rapid assessment and timely classification of patients with COVID-19-related pneumonia are very important. However, no scoring system has been designated for the rapid assessment and prognosis of patients with COVID-19. Such a system is urgently needed.

Objective: To explore the factors affecting mortality in patients with COVID-19 and to verify if the predictive value of the three rapid scoring scales [the Modified Early Warning Score (MEWS), Rapid Acute Physiology Score (RAPS) and Rapid Emergency Medicine Score (REMS)] that are commonly used in emergency departments can also be used for the prognostication of COVID-19 patients.

Methods: The study included adult patients diagnosed with COVID-19 in Wuhan, China from February 7 to March 7, 2020. Kaplan-Meier and Cox survival analyses were performed to identify the risk factors associated with COVID-19-related death. C-index analysis was used to evaluate the abilities of the three scoring scales and their combined score to predict the prognosis of COVID-19 patients.

Results: Older age, decreased lymphocyte count, increased respiration frequency, and low blood oxygen saturation level were identified as independent risk factors for mortality among patients with COVID-19. The Cox regression analysis demonstrated that MEWS, RAPS, and REMS had a statistically significant ability to predict mortality in COVID-19 patients $(P<0.05)$. Stats the $C$-index of MEWS, RAPS, REMS, and the combined score resulted $0.7,0.66,0.82$, and 0.83 respectively.

Conclusion: Patients with an old age, increased respiration frequency, low blood oxygen saturation level, and decreased lymphocyte count are at a high risk of COVID-19-related mortality. Moreover, our analysis revealed that the REMS had a better prognostic ability than the MEWS and RAPS when applied to COVID-19 patients. Our findings suggest that the REMS can be used as a rapid scoring tool for the early assessment of COVID-19 severity.

\section{Background}

Coronavirus disease 2019 (COVID-19) is caused by infection with the severe acute respiratory syndrome coronavirus-2 (SARS-CoV-2) [1]. As of April 2, 2020, more than 2,394,246 cases of COVID-19 and more than 164,527 related deaths have been reported in more than 200 countries and territories worldwide [2, 3]. In China, various hospitals have established specialized emergency departments as the first line of defense for COVID-19 patients. However, the wait times in these facilities are prolonged because of the large numbers of patients and relatively insufficient medical resources, and this situation increases the risk of nosocomial infection. Therefore, trends in the spread of COVID-19 and the outcomes of patients are related to the ability of a specialized emergency department to classify patients accurately [4]. Severe COVID-19 is associated with a high mortality rate, whereas relatively milder cases tend to resolve without intensive intervention. Therefore, the efficiency of COVID-19 patient classification could best be improved by applying appropriate scoring scales. These scales were designed as screening tools with the aim of reducing the time required to evaluate patients, and their use can greatly improve the quality of care and therapeutic effects and can thus reduce morbidity and mortality [5].

Various accurate and effective clinical scoring scales have been developed in recent years. For example, the Rapid Acute Physiology Score (Supplementary Material Table 1) is an abbreviated version of the Acute Physiology and Chronic Health Evaluation II (APACHE II) Scoring table, and several studies have found that this model can be used to evaluate patients' prognosis [6]. The Rapid Emergency Medicine Score (REMS), another commonly used clinical scale, comprises the variables contained in RAPS, as well as the patient's arterial oxygen saturation level and age (Supplementary Material Table 2). The effectiveness of the REMS model for the assessment of trauma patients has been confirmed in some studies [7-8]. The Modified Early Warning Score (MEWS) is also commonly applied to emergency patients (Supplementary Material Table 3) and can be used to detect potential disease-related changes in patients with severe disease at an early stage and enable measures to prevent deterioration [9]. However, no scoring scale has been designated specifically for the prognostic evaluation of COVID-19 patients. Therefore, this study aimed to assess and compare the prognostic values of the MEWS, RAPS, and REMS for the prognostic evaluation of COVID-19 patients in a specialized emergency department.

\section{Methods}

Study design. This was a retrospective analysis of data obtained from a database of patients admitted by the West China Hospital Medical Team during the anti-COVID-19 epidemic period in Wuhan. These patients were all admitted to critical wards in Wuhan People's Hospital. Initially, a survival analysis was applied to identify the patient-related risk factors for mortality. Next, the RAPS, REMS, and MEWS scoring scales were applied to the data, and their abilities to predict the prognosis of patients were assessed. 
The West China Hospital Institutional Review Committee approved the study and waived the requirement for informed consent from the study subjects due to the study design. The study complied with an international ethical guideline for human research, such as the Declaration of Helsinki. The accessed data were anonymized.

Settings and Subjects. This study included all adult patients diagnosed with COVID-19 between February 7 and March 7, 2020 ( $\mathrm{n}=79$ ). According to the diagnostic criteria of China's national health Committee for COVID-19, the disease degree of these patients was severe [10]. For all patients, the following data were retrieved from the database: basic information (sex, age, final diagnosis, and chronic diseases), vital signs (body temperature, heart rate, systolic and diastolic blood pressure, respiratory frequency), consciousness, oxygen saturation level and RAPS/REMS/MEWS scale scores, etc. The outcome variable was the patient's death or survival at discharge. The observation point for survival calculations was set as the discharge time of the last admitted patient.

Statistical analysis. The data analysis and mapping were conducted using the R-studio 7.0 and Prime 8.1. Continuous variables are presented as means \pm standard deviations, and categorical variables are described as composition ratios (\%). The Wilcoxon rank-sum test and Fisher's exact test were used to compare the continuous and categorical variables, respectively. The Kaplan-Meier (K-M) and Cox proportional hazards regression methods were used to perform survival analyses and univariate and multivariate survival analyses, respectively, to establish the relationships between potential predictive factors and mortality. After introducing three emergency rapid scoring scales, we calculated the score of each scale and their combined score, a Cox regression analysis was performed to establish a prediction model between the scores and the mortality rate. Concordance-index and time-dependent AUC was calculated to evaluate the abilities of the

RAPS, REMS, MEWS, and the combined score to predict mortality. For all analyses, a two-sided test was conducted with P-value less or equal to 0.05 considered to indicate statistical significance.

\section{Results}

Patients in the surviving and deceased groups had mean ages of $56.52 \pm 16.97$ and $75.05 \pm 12.94$ years, respectively, and this difference was significant $(\mathrm{P}<0.05)$. Moreover, the two groups differed significantly with respect to sex, systolic blood pressure, oxygen saturation level, white blood cell and lymphocyte counts, and the MEWS, RAPS REMS, and the combined scores (all P<0.05). In contrast, the two groups did not differ significantly in terms of the heart rate, diastolic blood pressure and body temperature $(P>0.05 ;$ Table 1$)$. 
Table 1

Difference between alive group and death group

\begin{tabular}{|c|c|c|c|}
\hline variable & $\begin{array}{l}\text { Alive } \\
n=60\end{array}$ & $\begin{array}{l}\text { Death } \\
n=19\end{array}$ & p-value \\
\hline $\begin{array}{l}\text { Gender } \\
\text { male }\end{array}$ & $25(41.6 \%)$ & 14(73.6\%) & 0.015 \\
\hline female & $35(58.3 \%)$ & $5(26.3 \%)$ & \\
\hline Age & $56.52 \pm 16.97$ & $75.05 \pm 12.94$ & $<0.001$ \\
\hline Heart rate & $83.60 \pm 15.01$ & $87.47 \pm 13.56$ & 0.248 \\
\hline Breathe & $19.83 \pm 2.068$ & $23.21 \pm 5.381$ & $0.006 *$ \\
\hline $\begin{array}{l}\text { Hypertension } \\
\text { no }\end{array}$ & $43(71.6 \%)$ & $13(68.4 \%)$ & 0.789 \\
\hline yes & $17(28.4 \%)$ & $6(31.6 \%)$ & \\
\hline Systolic pressure & $130.40 \pm 19.68$ & $143.30 \pm 24.85$ & $0.016 *$ \\
\hline Diastolic pressure & $80.40 \pm 10.75$ & $83.95 \pm 14.12$ & 0.347 \\
\hline Temperature & $36.59 \pm 0.45$ & $36.71 \pm 0.72$ & 0.388 \\
\hline oxygen saturation & $96.40 \pm 2.57$ & $86.53 \pm 12.46$ & $<0.001$ \\
\hline While cell count & $5.54 \pm 1.91$ & $11.57 \pm 9.79$ & 0.001 \\
\hline Lymphocyte count & $27.56 \pm 11.8$ & $8.86 \pm 9.48$ & $<0.001$ \\
\hline MEWS & $1.48 \pm 0.87$ & $2.37 \pm 1.53$ & $0.001 *$ \\
\hline RAPS & $0.80 \pm 1.31$ & $1.58 \pm 1.71$ & $0.03^{*}$ \\
\hline REMS & $3.90 \pm 2.98$ & $7.90 \pm 2.81$ & $<0.001 *$ \\
\hline Combined Score & $6.18 \pm 4.47$ & $11.85 \pm 5.13$ & $<0.001 *$ \\
\hline
\end{tabular}

Next, a K-M survival analysis and a univariate Cox regression analysis were performed to identify the variables that differed significantly between the surviving and deceased groups. Notably, the patient's age, sex, respiratory frequency, oxygen saturation level, lymphocyte count and chronic disease status were identified as significantly different between the groups $(P<0.05)$. We chose significant variables after UVA analysis into a subsequent multivariate Cox regression analysis. the patient's age, respiration frequency, oxygen saturation level and lymphocyte count were identified as independent risk factors for mortality $(P<0.05)($ Table 2$)$. The patients' survival outcomes classified by sex and chronic disease status are presented in Fig. 1a and b, respectively. 
Table 2

Survival-related factors based on the survival outcomes

\begin{tabular}{|llll|}
\hline Variables & UVA & & MVA \\
\cline { 2 - 4 } & Adjusted HR $(95 \% \mathrm{Cl})$ & P-value & Adjusted HR (95\% Cl) \\
\hline Age & $1.081(1.09-1.124)$ & $<0.001$ & $1.043(0.99-1.093)$ \\
\hline Sex & & & \\
\hline F & Reference & Reference \\
\hline M & $0.278(0.01-0.78)$ & $\mathbf{0 . 0 1 4}$ & $1.18(0.33-4.20)$ \\
\hline Respiration frequency & $1.236(1.125-1.359)$ & $<0.001$ & $1.17(1.02-1.34)$ \\
\hline Oxygen saturation level & $0.804(0.794-0.864)$ & $<0.001$ & $0.879(0.802-0.964)$ \\
\hline Lymphocyte count & $0.89(0.847-0.936)$ & $<0.001$ & $0.94(0.885-0.999)$ \\
\hline Chronic disease & & & 0.003 \\
\hline No & Reference & & 0.04 \\
\hline Yes & $2.94(1.15-7.53)$ & $\mathbf{0 . 0 2 2}$ & $0.341(0.103-1.125)$ \\
\hline Cls are presented as lower limit- upper limit ranges. & & \\
\hline Abbreviations: UVA, univariate analysis; MVA, multivariable analysis; HR, hazard ratio; $95 \%$ Cl, 95\% confidence interval \\
\hline
\end{tabular}

The Cox regression analysis demonstrated that MEWS, RAPS, REMS, and the combined score had a statistically significant ability to predict mortality in COVID-19 patients ( $P<0.05$; Table 3 ). Stats the $C$-index of MEWS, RAPS, and REMS resulted 0.7,0.66 and 0.82 respectively. Comparing to REMS, both MEWS and Combined Score have significantly less predictive ability on overall survival (both $\mathrm{P}<0.05)$. The Cindex of the combined score was 0.83 , which is not significantly better comparing to the C-index of REMS $(P>0.05)$.

Table 3

Cox regression analysis of MEWS, RAPS, and REMS scores on survival outcome.

\begin{tabular}{|llllll|}
\hline & HR & Low 95\%Cl & High 95\%Cl & P value & C-index \\
\hline MEWS & 1.8428 & 1.3619 & 2.4936 & $<0.0001$ & 0.70 \\
\hline RAPS & 1.4088 & 1.0696 & 1.8555 & 0.0147 & 0.66 \\
\hline REMS & 1.4783 & 1.2482 & 1.7508 & $<0.0001$ & 0.82 \\
\hline Combined Score & 1.228 & 1.1244 & 1.3412 & $<0.0001$ & 0.83 \\
\hline Besides the C-index for each feature prediction., & & \\
\hline
\end{tabular}

\section{Discussion}

COVID-19, a novel infectious disease caused by SARS-CoV-2 [10], was first identified in December 2019 in Wuhan, the capital of Hubei Province in China. Since then, the disease has spread globally, resulting in the ongoing 2019-20 COVID-19 pandemic [11-12]. The common symptoms of COVID-19 include fever, cough and dyspnea, while some patients may also present with muscle pain, sputum production, diarrhea, pharyngitis, anosmia, and abdominal pain. Although the majority of cases result in mild symptoms, some progress to viral pneumonia and multi-organ failure that particularly affects the lung [13-14]. Currently, COVID-19 is diagnosed mainly by viral nucleic acid detection tests and lung computed tomography scans. Although clinicians also use parameters such as the respiratory frequency, heart rate, body temperature, arterial oxygen partial pressure, and indexes such as the white blood cell count, CRP concentration, and APACHE II score to classify COVID-19 cases according to severity, these prognostic factors are not specific [15]. Moreover, the APACHE II scale is complex and requires a long time; therefore, it is not suitable for the rapid prognostic evaluation of a COVID-19 patient. The identification of prognostic factors that could indicate the severity of COVID-19 at an early stage would both improve patients' outcomes and reduce the mortality rate. As no existing scale is available specifically to determine the severity of COVID-19 at an early stage, we compared the abilities of three emergency rapid scoring scales and combined predictors to evaluate the prognosis of COVID-19 patients. Our results identified the REMS as the best performing scale in this population. 
SARS-CoV-2 infects host cells through interactions between the viral surface spike S protein and angiotensin converting enzyme 2 (ACE2) on the surfaces of host cell membranes. ACE2 is widely expressed in various tissues of human body, especially the alveolar epithelium, small intestinal epithelium, and vascular endothelial cells. Upon entering the host cell, the virus replicates, and is released and simultaneously stimulates the host immune defense responses [16-17]. Studies have observed normal or decreased white blood cell counts, decreased lymphocyte counts, and increased serum C-reactive protein, creatinine and uric acid concentrations in some patients with early-stage COVID$19[18,19]$. In the present study, the patient's age, respiratory frequency, systolic blood pressure, oxygen saturation level, and white blood cell and lymphocyte counts were found to differ significantly between subjects in the surviving and deceased groups. According to previous studies, underlying medical conditions such as hypertension, lung disease, diabetes, cancer, and immunocompromised status were important factors affecting the prognosis of COVID-19 [20-21]. Similarly, we found that in addition to age, respiratory frequency, lymphocyte count, and blood oxygen saturation level, the presence of a chronic underlying disease was an independent risk factor for mortality in COVID19 patients.

Of the scales evaluated in this study, the MEWS is an internationally recognized and effective early warning scoring method used to determine the severity of a disease condition [22-23]. The MEWS includes evaluations of the patient's body temperature, heart rate, systolic blood pressure, respiratory rate and consciousness. However, our study indicated that the MEWS yielded 0.70 predictive ability for the prognostic evaluation of COVID-19. The RAPS can be used to assess the mortality risk of patients in the ICU or those with severe trauma during transportation. This scale includes four parameters: blood pressure, pulse, respiratory frequency, and Glasgow coma scale (GCS) score. [24-26]. However, the predictive ability of the RAPS differs among disease types. In our research, the RAPS yielded only 0.66.

The REMS was first applied by Olsson in 2003 to predict the mortality of patients with severe disease in an emergency internal medicine department [7]. This scoring system is ideal for rapid prognostication in an emergency department, and it features the advantages of a simple collection of observed indicators, the provision of relatively reliable information, and the ability to enable an emergency department to determine a patient's prognosis at an early stage [27]. The REMS evaluate six indicators: heart rate, systolic blood pressure, respiratory frequency, GCS score, age, and oxygen saturation level. In our study, the REMS yielded a predictive ability of 0.82 as well as a significantly greater ability than those yielded by the RAPS and MEWS. These findings suggest that the REMS can be used as a rapid assessment tool for the prognostication of patients with COVID-19 in an emergency setting. We further determined that an old age, respiratory frequency and low oxygen saturation level are risk factors for mortality in COVID-19 patients. As the REMS evaluation index includes the three items, we speculate that this is why the REMS demonstrated a better prognostic ability in our study. We additionally evaluate the combined score of MEWS, RAPS, and REMS and assessed the ability of this combined score to predict the prognosis of patients. It yielded an ability of 0.83 . Although this represents a slight improvement over the results obtained with the REMS alone, the difference is not significant. Therefore, we do not recommend using this combination of three scoring systems when determining the prognosis of a COVID-19 patient.

This study was limited by a small sample size and a lack of details regarding the classification of chronic diseases, which made it difficult to determine which chronic diseases truly affect the prognosis of COVID-19 patients. Moreover, our analysis of the MEWS, RAPS and REMS revealed that not all of the included items were identified as independent risk factors affecting prognosis, and consequently none of the scales yielded prognostic ability higher than 0.9. Future studies should aim to expand the sample size, identify additional effective indicators and develop a new scale specifically for the evaluation of COVID-19 patients.

\section{Conclusion}

Patients with an old age, increased respiration frequency, low blood oxygen saturation level, and decreased lymphocyte count are at a high risk of COVID-19-related mortality. Moreover, our analysis revealed that the REMS had a better prognostic ability than the MEWS and RAPS when applied to COVID-19 patients. Our findings suggest that the REMS can be used as a rapid scoring tool for the early assessment of COVID-19 severity.

\section{List Of Abbreviations}




\begin{tabular}{ll} 
coronavirus disease 2019 & COVID-19 \\
\hline the Modified Early Warning Score & MEWS \\
\hline Rapid Acute Physiology Score & RAPS \\
\hline Rapid Emergency Medicine Score & REMS \\
\hline severe acute respiratory syndrome coronavirus-2 & SARS-CoV-2 \\
\hline Acute Physiology and Chronic Health Evaluation II & APACHE II \\
\hline angiotensin converting enzyme 2 & ACE2 \\
\hline Glasgow coma scale & GCS
\end{tabular}

\section{Declarations}

\section{Ethics approval and consent to participate}

The West China Hospital Institutional Review Committee approved the study and waived the requirement for informed consent from the study subjects due to the study design. The study complied with an international ethical guideline for human research, such as the Declaration of Helsinki. The accessed data were anonymized.

\section{Consent for publication}

Not applicable.

\section{Availability of data and materials}

The datasets analyzed during the current study are not publicly available but are available from the corresponding author on reasonable request.

\section{Competing interests}

The authors declare that they have no competing interests

\section{Funding}

This work was financially supported by Novel Coronavirus Project of West China Hospital of Sichuan University (HX2019nCoV063), the Strategic Priority Research Program of the Chinese Academy of Science (XDA23090502), and Health Emergency Project of Chengdu Health Commission (ZX2020005).

\section{Acknowledgments}

This work was financially supported by Novel Coronavirus Project of West China Hospital of Sichuan University (HX2019nCoV063), the Strategic Priority Research Program of the Chinese Academy of Science (XDA23090502), and Health Emergency Project of Chengdu Health Commission (ZX2020005).

\section{References}

1. Cucinotta D, and M Vanelli. "WHO declares COVID-19 a pandemic." Acta bio-medica: Atenei Parmensis.2020; 91.1: 157-160.

2. Cascella M, Rajnik M, Cuomo A, Dulebohn S C, Di Napoli R. Features, evaluation and treatment coronavirus (COVID-19). StatPearls Publishing 2020.

3. Coronavirus Update (Live): 2,394,246 Cases and 164,527 Deaths from COVID-19 Virus Outbreak - Worldometer". www.worldometers.info. Retrieved 19 April 2020.

4. Zhang Dan, Yu Yuan, Chen Junhua. Practice on precheck and triage management for SARS-CoV-2 infection in fever clinics in large general hospital. Nursing Research. 2020; 34(4):1-2.

5. Nogueira Lde S, Domingues Cde A, Poggetti RS, de Sousa RM. Nursing workload in intensive care unit trauma patients: analysis of associated factors. PLoS One. 2014;9(11): e112125. 
6. Rhee, Kenneth J., Charles J. Fisher Jr, and Neil H. Willitis. The rapid acute physiology score. The American journal of emergency medicine. 1987;5.4: 278-282.

7. Olsson T, Lind L. Comparison of the Rapid Emergency Medicine Score and APACHE II in Nonsurgical Emergency Department Patients. Academic Emergency Medicine. 2003;10(10):1040-8.

8. Olsson T, Terent A, Lind L. Rapid Emergency Medicine Score Can Predict Long-term Mortality in Nonsurgical Emergency Department Patients. Academic Emergency Medicine. 2004;11(10):1008-13.

9. J Gardner-Thorpe, N Love, J Wrightson, S Walsh, N Keeling. The value of Modified Early Warning Score (MEWS) in surgical in-patients: a prospective observational study. The Annals of The Royal College of Surgeons of England. 2006;88.6: 571-575.

10. National Health Commission. The Diagnosis and Treatment Plan of Novel Coronavirus (Version 6) . http://www.nhc.gov.cn/yzygj/s7653p/202002/8334a8326dd94d329df351d7da8aefc2/files/b218cfeb1bc54639af227f922bf6b817.pdf. Accessed Feb 19, 2020.

11. Coronavirus disease (COVID-19) Pandem. https://www.who.int/emergencies/diseases/novel-coronavirus-2019. Accessed Apr 10, 2020.

12. Hui DS, I Azhar E, Madani TA, Ntoumi F, Kock R, Dar O, et al. The continuing 2019-nCoV epidemic threat of novel coronaviruses to global health-The latest 2019 novel coronavirus outbreak in Wuhan, China. International Journal of Infectious Diseases. 2020; 91: 264-266.

13. Wu Zunyou, Jennifer M. McGoogan. Characteristics of and important lessons from the coronavirus disease 2019 (COVID-19) outbreak in China: summary of a report of 72314 cases from the Chinese Center for Disease Control and Prevention. Jama (2020).

14. Haibo Zhang, Josef M. Penninger, Yimin Li, Nanshan Zhong, Arthur S. Slutsky. Angiotensin-converting enzyme 2 (ACE2) as a SARS-CoV2 receptor: molecular mechanisms and potential therapeutic target. Intensive Care Med. 2020;46, 586-590.

15. Huang Chaolin, Wang Yeming, LiXingwang, Ren Lili, Zhao Jianping, Hu Yi, et al. Clinical features of patients infected with 2019 novel coronavirus in Wuhan, China. The Lancet. 2020; 395(10223): 497-506.

16. Hoffmann, Markus, et al. SARS-CoV-2 cell entry depends on ACE2 and TMPRSS2 and is blocked by a clinically proven protease inhibitor. Cell. 2020;181.2:271-280.

17. Y Wan, J Shang, R Graham, RS Baric, F Li. Receptor recognition by the novel coronavirus from Wuhan: an analysis based on decade-long structural studies of SARS coronavirus. Journal of virology. 2020; 94(7):127-147.

18. J Xie, N Covassin, Z Fan, P Singh, W Gao, G Li. Association Between Hypoxemia and Mortality in Patients With COVID-19. Mayo Clinic Proceedings. Elsevier. 2020.

19. X Luo, W Zhou, X Yan, T Guo, B Wang, H Xia, et al. Prognostic value of C-reactive protein in patients with COVID-19. MedRxiv. 2020.

20. N Tang, D Li, X Wang, Z Sun. Abnormal coagulation parameters are associated with poor prognosis in patients with novel coronavirus pneumonia. Journal of Thrombosis and Haemostasis. 2020;18.4:844-847.

21. .Dong Ji, Dawei Zhang, Jing Xu, Zhu Chen, Tieniu Yang, Peng Zhao, et al. Prediction for Progression Risk in Patients with COVID-19 Pneumonia: the CALL Score, Clinical Infectious Diseases. 2020; ciaa414.

22. K Salottolo, M Carrick, J Johnson, M Gamber. A Retrospective Cohort Study of the Utility of the Modified Early Warning Score for Interfacility Transfer of Patients with Traumatic Injury. BMJ Open, 2017, 7 (5): e016143.

23. CP Subbe, M Kruger, P Rutherford, L Gemmel. Validation of a Modified Early Warning Score in Medical Admissions. Q JMed. 2001;94 (10) :521-526.

24. BF Imhoff, NJ Thompson, MA Hastings, N Nazir. Rapid Emergency Medicine Score (REMS) in the trauma population: a retrospective study. BMJ Open. 2014;4(5): e004738.

25. SK Hung, CJ Ng, CF Kuo, ZNL Goh, LH Huang, CH Li,et al. Comparison of the Mortality in Emergency Department Sepsis Score, Modified Early Warning Score, Rapid Emergency Medicine Score and Rapid Acute Physiology Score for predicting the outcomes of adult splenic abscess patients in the emergency department. PLoS One. 2017;12(11):e0187495.

26. OLSSON, Thomas; TERÉNT, Andreas; LIND, Lars. Rapid Emergency Medicine Score: a new prognostic tool for in-hospital mortality in nonsurgical emergency department patients. Journal of internal medicine. 2004;255.5: 579-587.

27. M Bulut, H Cebicci, D Sigirli, A Sak, O Durmus. The comparison of modified early warning score with rapid emergency medicine score: a prospective multicentre observational cohort study on medical and surgical patients presenting to emergency department. Emergency Medicine Journal. 2014;31.6: 476-481.

\section{Figures}




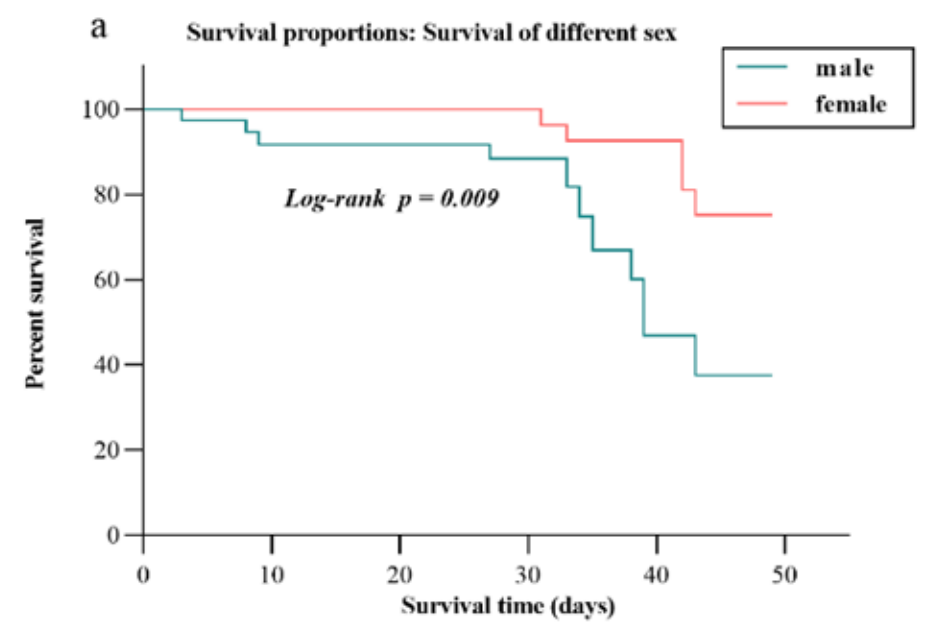

b Survival proportions: Survival of chronic disease and non-chronic disease

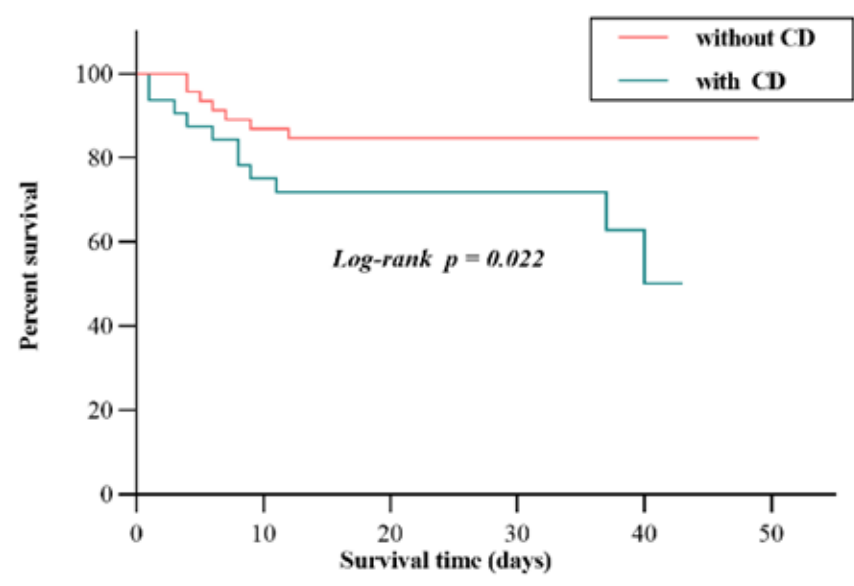

Figure 1

Kaplan-Meier survival estimates according to $a$. sex and b. chronic disease status

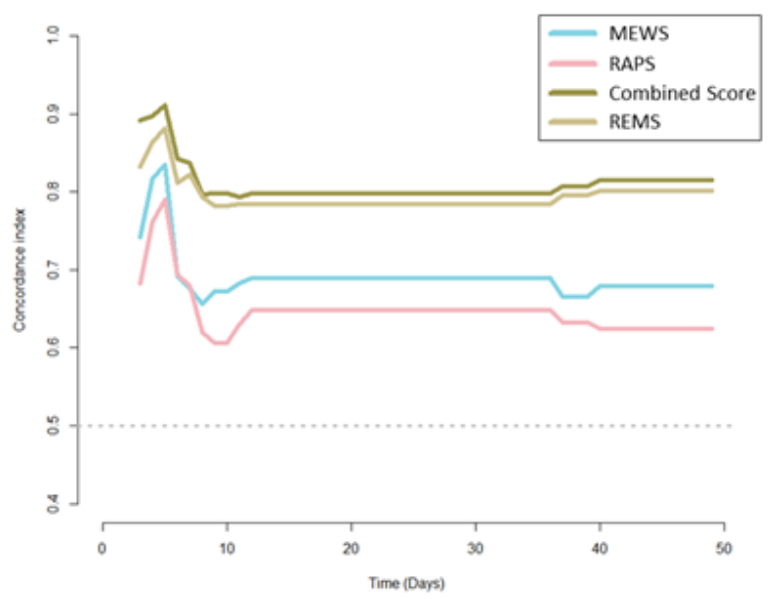

Figure 3

Time-dependent AUC of MEWS, RAPS, REMS and Combined Score. 